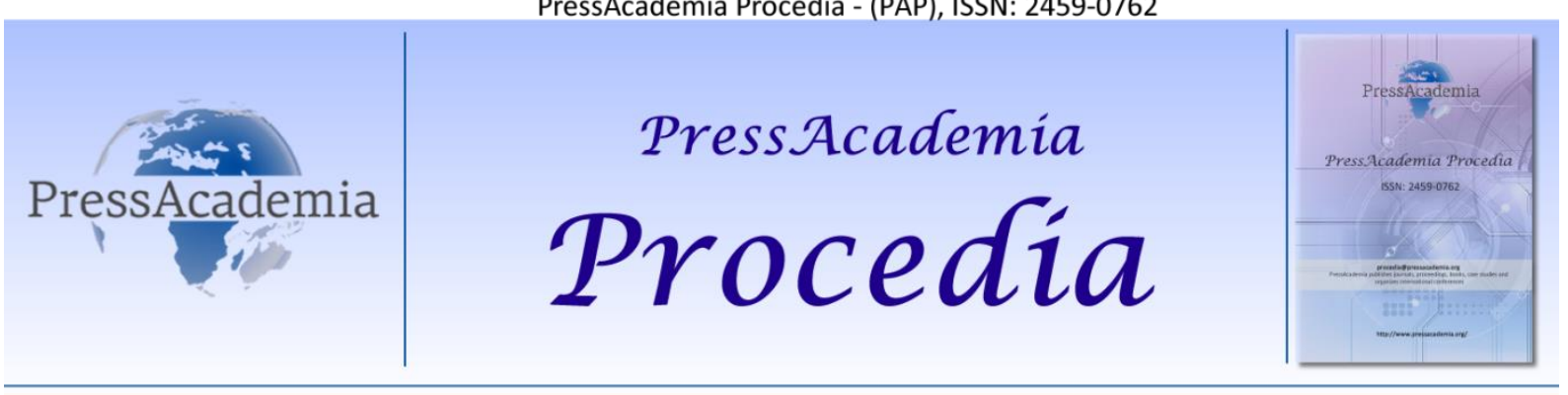

2nd World Conference on Technology, Innovation and Entrepreneurship

May 12-14, 2017, Istanbul, Turkey. Edited by Sefer Şener

\title{
DISPLAYING OF DATA'S OF DEVELOPED ENVIRONMENTALLY INTELLIGENT SYSTEMS USING WIRELESS SENSOR NETWORKS ON ANDROID PLATFORMS
}

\author{
DOI: 10.17261/Pressacademia.2016.570 \\ PAP-WCTIE-V.5-2017(8)-p.47-51
}

\section{Murat Dener ${ }^{1}$}

${ }^{1}$ Gazi University, muratdener@gazi.edu.tr

\begin{abstract}
Wireless Sensor Networks consist of large number of tiny sensor nodes. Each sensor node has communication, sensing and computing capabilities. These sensor nodes perform the environmental measurement tasks with the cooperation. Intelligent systems can be developed using these sensor nodes. Also, the number of mobile applications used in the display of intelligent systems has increased rapidly in recent years. As a result of this rapid increase, displaying and control of data over these mobile platforms have also begun. In this study, a wireless sensor network that detects the environmental data has been established using WiSeN sensor nodes. In addition, a mobile application has been developed to monitor the data's. This study brings innovation to the monitoring of environmental data's.
\end{abstract}

Keywords: Wireless sensor networks, android platforms, environmental data's

\section{INTRODUCTION}

It gets harder and harder every day to count the number of the technologies coming into our lives. The concept of mobile has also come into our lives as a result of these developments. Most people think of smart phones when they hear about this concept. Apart from smart phones, many other devices serving for far different areas are also being produced. All mobile devices fall into mobility. Mobile phones have made the concept of mobile get about. These devices used to be utilised for searching and messaging only. But nowadays, many transactions such as downloading from the Internet, making modifications on them and forwarding to someone else are also performed. It would be a big mistake to consider electronic devices, as the software inside is not visible, as a hardware only. As far as the mobile phones are concerned, each one of them has a unique operating system. As per the report of IDC (2017), the Android operating system dominates $84 \%$ of the market in the third quarter of 2014. Therefore, the Android operating system is the one with most users. DiMarzio (2008) states that an android operating system is an open source coded mobile operating system created using Linux 2.6 kernel. However, unless it has got applications on it, it resembles a car with no engine. It should be noted that when there is no applications installed, not even search can be made. Every service used on Android operating system is an application.

Wireless Sensor Networks are created by means of connecting many sensor nodes over a wireless connection. These nodes can make happen data communication from one region over the other in a network they create in which they communicate with each other. The data collected from the wireless sensor networks are sent to the database by means of the Internet Module. The Android application created takes the data that have been sent from the data base using web services. The data received are displayed on the Android interface. For instance, the values of temperature, humidity, light and such can be displayed on the interface of the Android instantly using a sensor network infrastructure.

The paper is organized as follows. In the second part of the study information on wireless sensor networks and the sensor node are presented. In the third part, making of the application is explained in detail. In the last part, the conclusion is presented. 


\section{WIRELESS SENSOR NETWORKS}

Akyildiz et al. (2002) state that the progresses in the low-cost sensor architectures have made the Wireless Sensor Networks a new and known research area. Chong et al. (2003) state that Wireless Sensor Networks consist of small, limitedcapacity sensor nodes. Lin et al. (2004) state that each sensor node has the ability to calculate, detect and communicate. When the energy of the sensor nodes is exhausted, the life of the node ends. Because of this, low power listening techniques are working within the sensor nodes. The sensor nodes send the information they have detected through cooperation to the gateway node. The information from the gateway node is displayed on the web or mobile platforms. Because of these features, Wang et al. (2006) state that they can be used in a wide variety of areas. Area of usage of the Wireless Sensor Networks is increasing with each passing day. Wireless Sensor Networks can be utilised in many fields including; guarding of battle fields, monitoring of enemy moves, reconnoitring of the fields, monitoring of military and personnel vehicles, monitoring of friendly forces, and determination of the speed and location of the targets in Military applications, in the environmental applications requiring determination of weather forecast, air pollution, monitoring of natural disasters such as floods, earthquakes forest fires, monitoring of agricultural activities and monitoring of animal farms, in medical applications consisting of determining the location of the doctors in the Hospital, monitoring of the conditions of the patients, guarding of the elderly and following some medical parameters, in commercial applications such as monitoring and determining of vehicles, monitoring of power lines, following of children by their parents, checking of lightings, checking of traffic lights and fire alarm systems and in home automation systems requiring smart home application and building security systems. The WiSeN sensor node used in study is shown in Figure 1.

Figure 1: WiSeN Sensor Node

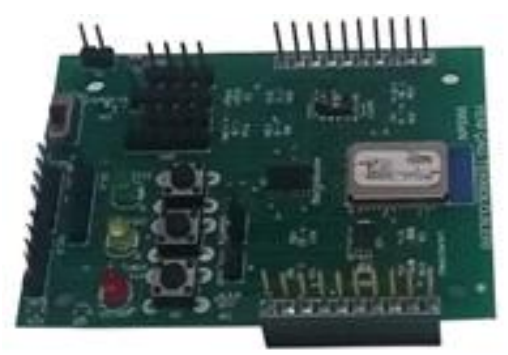

General Characteristics of the WiSeN sensor node (2017): Zigbee IEEE 802.15.4, 2.4 GHz ISM band, CC2530 Radio Module, MSP430 Microcontroller, Low Power Consumption, Low Cost, To integrate the desired sensor, To acquire features to sensor node with C language, Programming via the USB Interface, Remote access to each sensor node, Self-organizing, The advantage of gateway node (Server, Sms, Call), Following datas over WEB, Mobile (Android, iOS) platforms, Ability to communicate with different wireless sensor nodes.

\section{APPLICATION}

A database is a space in which related data are brought together. With the use of these spaces the access to these data are lot easier. And the web services act as a door for these data to be accessed from outside. Any application requesting to get data from the database is connected to the web service first. Then whatever data the web service is supposed to receive is taken and sent to the application. In the web service created within the framework of this study PHP language has been used. The data are sent and received in JSON. JSON (JavaScript Object Notation) is a text format that facilitates structured data exchange between all programming languages. The application needed in order for the environmental data received from Wireless Sensor Networks to be displayed in Android Platforms comprises of four sections. These are: Application Splash Screen, Registered Users Entry Screen, Category Selection Screen, Screen for Scenarios.

\subsection{Application Splash Screen}

This is the section where the logo and slogan of the application is located. When the application is started, this section runs first. It stays on for a while. This duration can be determined by the developer. The application splash screen is shown in Figure 2. 
Figure 2: Application Splash Screen

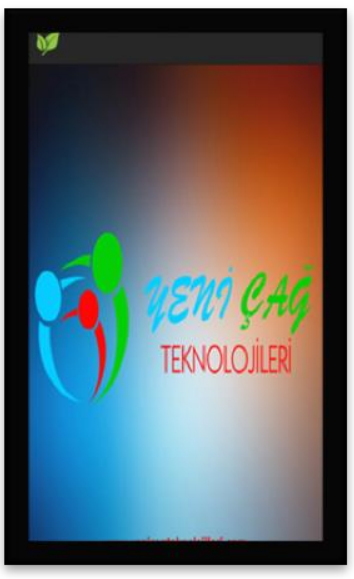

\subsection{Registered Users Entry Screen}

It is the second screen coming up. The users enter their user names and passwords. If an earlier session has been initiated, this screen is not displayed. The user name and the password is encrypted in JSON and is asked to the web service of the server defined in a POST query. Following the checking of user name and password by the web service, the "User ID", the "User Name" and the "User Name and Surname" are sent to the application. The application splits these data and forwards to the required configurations in which they are to be used. The registered users entry screen is shown in Figure 3.

\section{Figure 3: Registered Users Entry Screen}

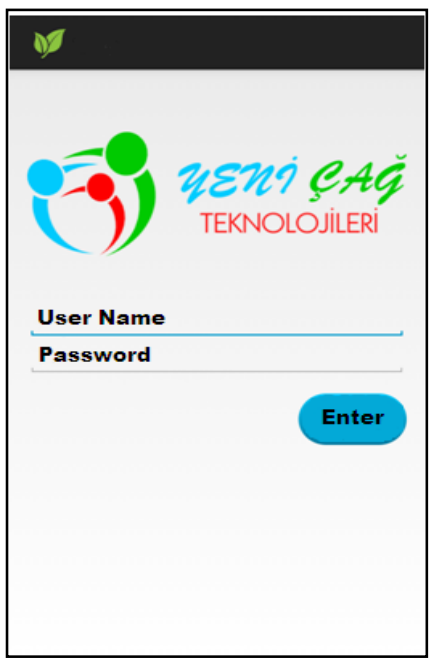

\subsection{Category Selection Screen}

This section is divided into two as left and right. These sections are called "frames". In the right frame are the categories. The contents of the categories are in shown in the left frame. For instance; when the "Temperature" category on the right frame is selected, the temperature data is displayed in the left frame. When any of the categories in the right frame is selected, the application pulls the data from the web service and displays the required data on the left frame. The data coming to this section is transiently updated. For instance, the temperature values sensed by the sensor nodes will transiently be displayed in this section. As many categories as desired can be placed in the right frame. If the categories are represented in symbols, the visuality of the interface would be more attractive to the end user. More than one button can be added on the action bar in this section. These can be logging out and scenarios. When the log out button is hit, the session of the user is ended and the session opening screen is displayed. When the scenarios button is hit, the screen with scenarios is opened up. Category selection screen is shown in Figure 4. 


\section{Figure 4: Category Selection Screen}

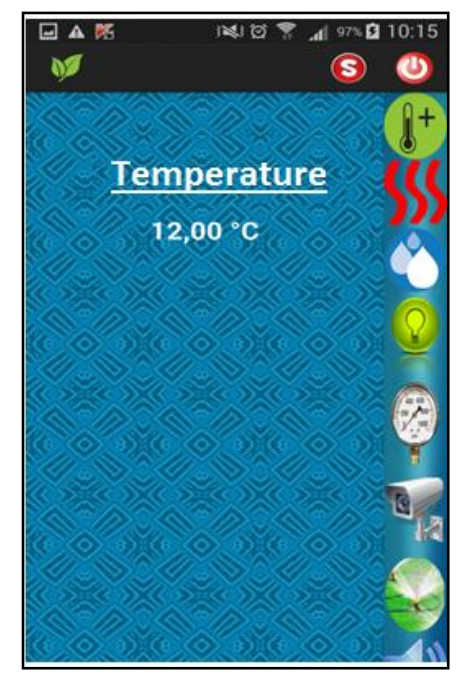

\subsection{Screen for Scenarios}

In order for the users to enter into the section for scenarios, they select scenarios in the action bar. As a result of the selection scenarios screen will be entered into. There is a spinner object in this screen (Figure 5). With this object, the scenarios can be viewed in a list. The data for this list extracted from the database. When w scenario is selected from the list, the list for selection is wiped off from the screen, as a result of the feature of the spinner, and the features of the scenario selected is brought up to the screen (Figure 6). For instance, with a scenario made up in the temperature category, the application can be instructed to send an SMS to the user if the temperature goes over a pre-defined value.

\section{Figure 5: Screen for Scenarios 1}

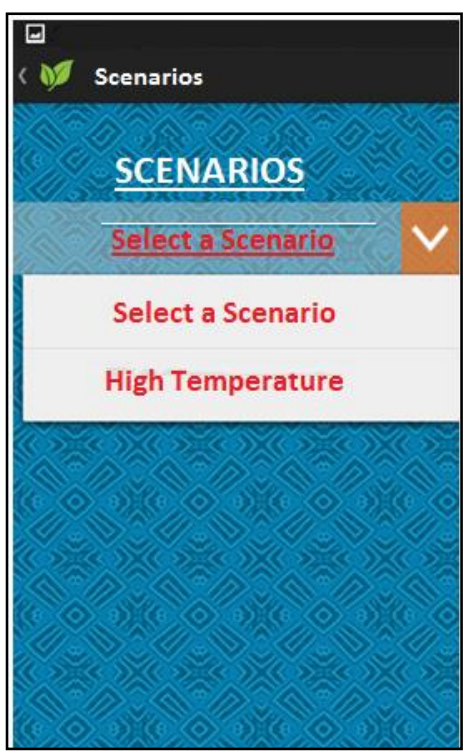




\section{Figure 6: Screen for Scenarios 2}

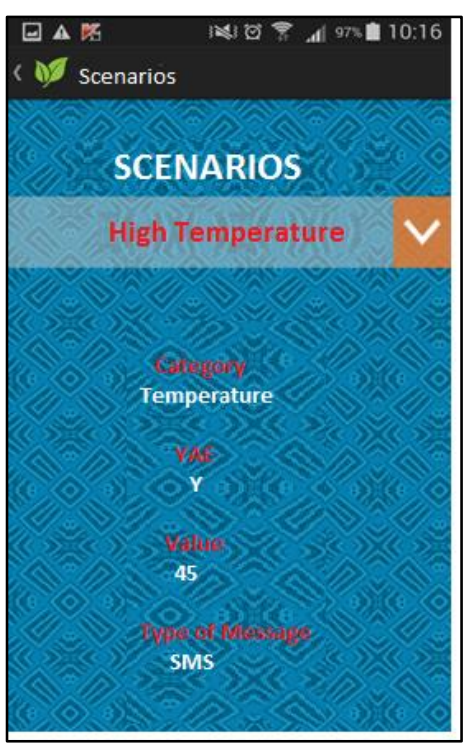

\section{CONCLUSION}

In this work, data from wireless sensor nodes was monitored on the Android platform. The Android application created in the methods mentioned can be run on all devices using Android operating system. As the application gets in connection with the web service, it does not get affected by the changes made in database. Therefore, when there is change in the database section of an application uploaded in the application market, the application can run without needing for an upgrade.

\section{REFERENCES}

Akyildiz, I.F., Su, W., Sankarasubramaniam, Y., Cayirci, E. 2002, “A survey on sensor networks”, IEEE Communications Magazine, 40(8), 102114.

Chong, C.Y., Kumar, S.P, 2003, “Sensor Networks : Evolution, opportunities, and challenges”, Proc IEEE, Vol 91, No 8, $1247-1256$.

DiMarzio, J.F. 2008, Androıd A Programmers Guıde, McGraw Hill Professional.

Lin, R., Wang, Z., Sun, Y. 2004, "Energy Efficient Medium Access Control Protocols for Wireless Sensor Networks and Its State-of-Art", IEEE, pp 669-674.

New Age Technologies 2017, http://yenicagteknolojileri.com/portfolio-item/wsn-sensor-dugumu/.

Smartphone OS Market Share 2017, IDC [Online]. Available: http://www.idc.com/prodserv/smartphone-os-market-share.jsp.

Wang, y., Attebury, G., Ramamurthy, B. 2006, "A Survey Of Security Issues In Wireless Sensor Networks" , IEEE Communications Surveys \& Tutorials, Volume 8, No. 2, 2nd Quarter. 